\title{
Impact of information intervention on stochastic hepatitis B model and its variable-order fractional network
}

\author{
Anwarud Din ${ }^{1} \mathbb{D}$, Yongjin Li1 ${ }^{1, a}$, Abdullahi Yusuf ${ }^{2,3}$, Jinping Liu ${ }^{4}$, and Ayman A. Aly ${ }^{5}$ \\ 1 Department of Mathematics, Sun Yat-sen University, Guangzhou 510275, People's Republic of China \\ 2 Department of Computer Engineering, Biruni University, Istanbul, Turkey \\ 3 Department of Mathematics, Federal University Dutse, Jigawa, Nigeria \\ 4 Hunan Provincial Key Laboratory of Intelligent Computing and Language, Information Processing, Hunan Normal \\ University, Changsha 410081, China \\ 5 Department of Mechanical Engineering, College of Engineering, Taif University, P.O. Box 11099, Taif 21944, Saudi Arabia
}

Received 15 April 2021 / Accepted 13 January 2022 / Published online 4 February 2022

(C) The Author(s), under exclusive licence to EDP Sciences, Springer-Verlag GmbH Germany, part of Springer Nature 2022

\begin{abstract}
This paper aims at analyzing the dynamical behavior of a SIR hepatitis B epidemic stochastic model via a novel approach by incorporating the effect of information interventions and random perturbations. Initially, we demonstrate the positivity and global existence of the solutions. Afterward, we derive the stochastic threshold parameter $\mathbf{R}_{\mathbf{s}}$, followed by the fact that this number concludes the transmission of hepatitis B from the population. By increasing the intensity of noise, we get $\mathbf{R}_{\mathbf{s}}$ less than one, inferring that ultimately hepatitis B will lapse. While decreasing the intensity of noise to a sufficient level, we have $\mathbf{R}_{\mathbf{s}}>1$. For the case $\mathbf{R}_{\mathbf{s}}>1$, adequate results for the presence of stationary distribution are achieved, showing the prevalence of hepatitis $\mathrm{B}$. The present study also involves the derivation of the necessary conditions for the persistence of the epidemic. Finally, the main theoretical solutions are plotted through simulations. Discussion on theoretical and numerical results shows that utilizing random perturbations and information interventions have a pronounced impact on the syndrome's dynamics. Furthermore, since most communities interact with each other, and the disease spread rate is affected by this factor, a new variable-order fractional network of the stochastic hepatitis B model is offered. Subsequently, this study will provide a robust theoretical basis for comprehending worldwide SIR stochastic and variable-order fractional network-related case studies.
\end{abstract}

\section{Introduction}

Human history reveals that infectious diseases are the real source of threat and adversely affect an individual's health and socioeconmic status. Referring to the H7N9 bird flu (the first novel sub-type of influenza virus) originated from Anhui and Shanghai in 2013 [1,2]. The H7N9 symptoms of the disease include high fever, particularly at the initial days of the infection. The disease continues to spread, and till now, the disease caused thousand of infections and hundreds of deaths. We also know that the recent COVID-19 outbreak (announced pandemic by the WHO) which almost affected the entire world and caused millions of infections and more than $1.17 \mathrm{M}$ deaths [2]. With the occurrence of infectious diseases, people from all sectors start thinking of treating and preventing the disease at the earliest. Government and policymakers are spending massive budgets on the treatment and control of such infectious diseases. To make mathematical or other strategies to

\footnotetext{
a e-mail: stslyj@mail.sysu.edu.cn (corresponding author)
}

control the disease [3-5], so the very first intervention of the health officials is awareness about the disease $[6,7]$. With the help of high awareness and health education through media coverage, the disease may be prevented or at least one can delay its occurrence $[8,9]$. For example, during the $\mathrm{H} 7 \mathrm{~N} 9 \mathrm{flu}$, media played an active role in reporting infections, deaths, symptoms, control measures, and many other features of the disease on daily basis [10]. In the present COVID-19, media are playing more active role and at every movement, people are aware of the disease' status both in and outside of the country [11]. Researchers suggest that the majority of the infectious disease could be significantly controlled and the infection rate may be drastically reduced with the help of information coverage. There are many diseases which could be cured by imposing both pharmaceutical and non-pharmaceutical intervention strategies [30]. Spread of some diseases can be controlled with psychotherapy, because majority of infections are directly connected with an individual mind [13]. It is observed that psychological counseling in a positive sense will 
generate positive emotions which is important in resetting health activities and controlling epidemics $[13,14]$. In conclusion, we can argue that information interventions like health educations, psychological therapy, and media coverage may be considered.

It is interesting that good psychological advice can offer positive feelings to produce a negative impact of negative feelings caused by the perception of a negative emotion on the disease $[13,14,30]$. Infectious disease control and the development of healthy lives are ultimately boosted with the immediate effect of information [15]. Therefore, the interference of information (media reporting, wellbeing Psychological intervention, education) as a type of non-drug treatment is really relevant [9].

The mathematical formulation is a valuable method for explaining the dynamics of different outbreaks. A variety of ecologists, as well as mathematicians, have investigated the spread of mutual transmissible diseases with the help of different models describing the epidemics for understanding and monitoring. It is very commonly used to figure out the transmission as well as optimization of altered infectious diseases by models in term of various mood, i.e., deterministic epidemic models [20-22], fractional epidemic models [16-19], stochastic epidemic models [23-25], and age-structure models $[26,27]$. Fractional epidemic models [28-30]. In the case of infectious disease modeling, the more appropriating one as the stochastic modeling [31-34], due to it offer an extra degree of realistic comparable to its deterministic model compartments. It is also investigated that the stochastic-based models provide more output rather than deterministic ones as we may construct the division of the predicted results by running a stochastic model many times, while deterministic model, on the other hand, will give only one predicted value [35-40]. It has also been taken with keen interest that many studies are investigated by considering the intervention with the help of the information in the infectious diseases modeling. For example, Joshi [41] considered SIR as a model for infectious diseases based on intervention with information and found that information could decrease infection rates. In addition, the influence of information on vaccination has been studied by Buonomo et al. [42], with the help of SEIR model. Similarly, another study has been performed to investigate the effects of information as well as to forecast the control of disease [43]. More recently, the extinction as well as the persistence analysis of an epidemic model for hepatitis B have been performed by Khan and their co-authors [44]. However, to the best of our knowledge, no one incorporates HBV epidemic model with information intervention as well as variable-order fractional network. We formulate a new stochastic system in mathematical form to discuss the hepatitis B dynamics under the effect of information with random perturbations. We study the qualitative analysis of the global results and show that the solution satisfied the properties of uniqueness. We also perform the extinction analysis to obtain the conditions for extinction. Moreover, the analysis of persistence will be also investigated. Then, we will study the stationary distribution for the proposed model and then simulate the model to verify the analytical findings.

Also, in the current study, a new variable fractionalorder network is proposed for modeling of hepatitis B. By studying the factional order [45-56] or variable fractional-order network, we aim to consider the impact of the interaction between different communities on the disease rate in different societies. The relationships and travels between two countries, for instance, will definitely affect the rate of diseases in both of them. Hence, in this study, a network model is proposed. Also, to take advantage of fractional calculous, the model on the network is supposed to be fractional, which is the generalized form of integer model. In brief, by proposing this fractional-order network model, we hope to obtain more accurate results.

Organization of the paper: Sect. 2 is devoted to the formulation with biological feasibility. We show that the model preserves the properties of existence and uniqueness in Sect. 3, during the extinction with sufficient condition of stationary distribution as well as for persistence shown in Sects. 4, 6 and 5, respectively. Finally, the model results have been verified with the help of numerical simulations in Sect. 7. In Sect. 8, we show variable-order fractional network for the proposed model. The work has been finished in Sect. 9 with a brief conclusion.

\section{Model formulation}

Many authors and researchers have investigated by taking data about intervention into sequence infectious endemic systems like SIR, TB, HBV, and SEIR [4143], and they realized that availability of data can minimize the level of infected cases. In our manuscript, we proposed an SIR epidemic model corresponding to hepatitis B along with the effect of stochastic and information intervention to investigate the dynamics of hepatitis B virus disease. Our newly formulated SIR hepatitis $B$ virus model consists of susceptible, infected, recovered, as well as information intervention compartments. The following is our proposed SIR hepatitis B epidemic model:

$$
\begin{aligned}
& \frac{\mathrm{d} S(t)}{\mathrm{d} t}=\Lambda-\beta S(t) I(t)-\mu_{2} m Z(t) S(t)-\left(v+\mu_{0}\right) S(t), \\
& \frac{\mathrm{d} I(t)}{\mathrm{d} t}=\beta I(t) S(t)-\left(\mu_{0}+\gamma_{1}+\mu_{1}\right) I(t), \\
& \frac{\mathrm{d} R(t)}{\mathrm{d} t}=\gamma_{1} I(t)+\mu_{2} m Z(t) S(t)+v S(t)-\mu_{0} R(t), \\
& \frac{\mathrm{d} Z(t)}{\mathrm{d} t}=\frac{d I(t)}{(1+h I(t))}-a Z(t),
\end{aligned}
$$

where $S(t), I(t)$, and $R(t)$ symbolized by susceptible class, infected class, and recovered class, and $Z(t)$ represents the density of data availability in the of infected classes in the society. While, the model included parameters are summarized in the following list and assumed parameters will be positive: 
- $\Lambda$ : Per capita new born susceptible individuals.

- $\mu_{0}$ : Natural death rate.

- $\mu_{1}$ : HBV disease death rate.

- $\beta$ : The infected rate of susceptible.

- $v$ : Vaccination rate of HBV.

- $\mu_{2}$ : The intensity response rate.

- $\gamma_{1}$ : The rate of recovering.

- $m$ : Intervention information rate through which the behaviors of some individuals may changed their.

- $d$ : Represents information rate.

- $h$ :The constant of "Saturation"

- $a$ : The decreasing rate of information.

We know the "basic reproduction number" $\mathcal{R}_{0}$ of model (1) is $\mathcal{R}_{0}=\frac{\beta \Lambda}{\left(\mu_{0}+\gamma_{1}+\mu_{1}\right)\left(v+\mu_{0}\right)}$. The model (1) has two equilibrium points.

Disease-free equilibrium: If $1<\mathcal{R}_{0}$, then the model (1) will have their free equilibrium point (DEE) is denoted by $E_{1}=\left(S_{1}, I_{1}, R_{1}, Z_{1}\right)=\left(\frac{\Lambda}{v+\mu_{0}}, 0, \frac{v \Lambda}{\mu_{0}\left(v+\mu_{0}\right)}, 0\right)$, and the $E_{1}$ is asymptotically and globally stable.

Endemic equilibrium: If $1<\mathcal{R}_{0}$, then the system (1) exists an endemic equilibrium (EE) which is denoted by $E_{2}=\left(S_{2}, I_{2}, R_{2}, Z_{2}\right)$, and the $E_{2}$ is globally asymptotically stable.

Many researchers have defined and provide various random fluctuations for different diseases [57-59]. Among them is the well-known approach of fluctuating parameter in the considered system around an average value because of the consistent oscillation in the different environments $[60,60,61]$. It is clear that system (1) has the properties of a deterministic situation of modeling. Really, safe white noising will has a much more affect on infected population $[23,33,34,69]$, and the scholars pointed out that stochastic noising will bring changes in the basic number of reproduction for such type of disease. For the effect of environment noise, we may consider that the environment affect on different individuals is studied by the perturbations due to stochastic and it is related by proportionality to every compartment $S, I, R$. We used the standard Brownian motions is symbolized by $B_{1}(t), B_{2}(t)$ and $B_{3}(t)$ along with the intensities $\eta_{1}, \eta_{2}$ and $\eta_{3}$. Obviously, the motion due to Brownian will obey the fundamental postulates of $B_{1}(0)=B_{2}(0)=B_{3}(0)=0$. Then, we can write the the type of deterministic model (1) in stochastic style is given as

$$
\begin{aligned}
\mathrm{d} S(t)= & {\left[\Lambda-\mu_{2} m Z(t) S(t)-\beta S(t) I(t)-\left(v+\mu_{0}\right) S(t)\right] \mathrm{d} t } \\
& +\eta_{1} S(t) \mathrm{d} B_{1}(t), \\
\mathrm{d} I(t)= & {\left[\beta I(t) S(t)-\left(\mu_{0}+\gamma_{1}+\mu_{1}\right) I(t)\right] \mathrm{d} t } \\
& +\eta_{2} I(t) \mathrm{d} B_{2}(t) \\
\mathrm{d} R(t)= & {\left[\gamma_{1} I(t)+\mu_{2} m Z(t) S(t)+v S(t)-\mu_{0} R(t)\right] \mathrm{d} t } \\
& +\eta_{3} R(t) \mathrm{d} B_{3}(t) \\
\mathrm{d} Z(t)= & {\left[\frac{\mathrm{d} I(t)}{(1+h I(t))}-a Z(t)\right] \mathrm{d} t . }
\end{aligned}
$$

\section{Qualitative analysis of positive solution}

In the given part, we study the the properties of obtain solution of the problem (2). Before discussion, we introduce some basic concept related to our main study. For this let $\left(\Omega,\left\{F_{t}\right\}_{t \geq 0}, P\right)$ i.e., completely probabilistic space which may satisfy the condition of $X(t)=$ $(S(t), I(t), R(t), Z(t)),|X(t)|=\left(S^{2}(t)+I^{2}(t)+R^{2}(t)\right.$ $\left.+Z^{2}(t)\right)^{\frac{1}{2}}$, and $\mathbb{R}_{+}^{b}=\left\{x \in \mathbb{R}^{b}: \chi_{i}>0, i=1,2, \ldots, b\right\}$, where $\left\{F_{t}\right\}_{t \geq 0}$ is the filtration. We also consider a differential equation due to stochastic with $b$ dimensions as

$$
\mathrm{d} x(t)=f(t, x(t)) \mathrm{d} t+g(t, x(t)) \mathrm{d} B(t) \quad t \geq t_{o},
$$

with $x(t(0))=x_{0}$, where $x(0) \in \mathbb{R}^{d}$ and $B(t)$ is the standard $m$-dimensional Brownian motion. The linear operator $\mathcal{L}$ for the above equation is defined by

$$
\begin{aligned}
\mathcal{L}= & \frac{\partial}{\partial t}+\sum_{i=1}^{d} f_{i}(x, t) \frac{\partial}{\partial x_{i}} \\
& +\frac{1}{2} \sum_{i, j=1}^{d}\left[g^{T}(x, t) g(x, t)\right]_{i j} \frac{\partial^{2}}{\partial x_{i} \partial x_{j}} .
\end{aligned}
$$

Similarly, if $\mathcal{L}$ applied on $V$, and $V \in C^{2,1}\left(\mathbb{R}^{d} \times\right.$ $\left.\left[t_{0}, \infty\right) ; \mathbb{R}_{+}\right)$, then

$$
\mathcal{L} V=V_{t}+V_{x} f+\frac{1}{2} \operatorname{trace}\left[g^{T} V_{x x} g\right] .
$$

Moreover

$$
\begin{aligned}
& V_{t}=\frac{\partial V}{\partial t}, \quad V_{x}=\left(\frac{\partial V}{\partial x_{1}}, \ldots, \frac{\partial V}{\partial x_{d}}\right)^{T}, \\
& V_{x x}=\left(\frac{\partial^{2} V}{\partial x_{i} \partial x_{j}}\right)_{d \times d} .
\end{aligned}
$$

By generalized formula of Itô, it can be written as

$$
\mathrm{d} V=\mathcal{L} V \mathrm{~d} t+V_{x} g \mathrm{~d} B(t) .
$$


Now, with the help of above basic concepts, we are going to discuss the main result related to the existence and uniqueness analysis. To get these result, we have a bounded set $\Gamma$ as given under

$$
\begin{aligned}
& \Gamma=\{(S, I, R, Z) \in R_{+}^{4}: \frac{\Lambda}{\mu_{0}+\mu_{1}} \leq S+I \\
&\left.+R \leq \frac{\Lambda}{\mu_{0}}, 0 \leq Z \leq \frac{d \Lambda}{a\left(\mu_{0}+h \Lambda\right)}\right\} .
\end{aligned}
$$

Theorem 1 The solution of system (2), i.e., $(Z(t)$, $R(t), I(t), S(t))$ will be one for any $0 \leq t$ and for initial value $(Z(0), R(0), I(0), S(0),) \in R_{+}^{4}$, it will remain in $R_{+}^{4}$ with probability 1 , namely, $(S(t), I(t), R(t), Z(t)) \in$ $R_{+}^{4} \forall 0 \leq t$.

Proof Clearly, the coefficient are continuous locally locally lipschitz for $(Z(0), R(0), I(0), S(0))$ in $R_{+}^{4}$, which show that the solution $(Z, R, I, S)$ is unique and local in the interval $\left[0, \tau_{e}\right)$ at any $t$, where the explosion time is symbolized by $\tau_{e}$ (see for more detail $[23,31,63]$ ). If we can prove $\tau_{e}=\infty$ implies that the output is of global form. Take $k_{0}$ is a constant and assume that is nonnegative, and be a large number, so that $Z(0), R(0)$, $I(0)$ and $S(0)$ lies in $\left[k_{0}, \frac{1}{k_{0}}\right]$. Then, define $k_{0} \leq k$ and

$$
\begin{aligned}
\tau_{k}= & \left\{t \in\left[0, \tau_{e}\right): \min \{Z(t), R(t), I(t), S(t)\}\right. \\
& \leq \frac{1}{k} \text { or } \max \{Z(t), R(t), I(t), S(t) \geq 0\} .
\end{aligned}
$$

Now, define $\inf \Phi=\infty$, as $\Phi$ is the empty set. Here, $\tau_{k}$ is increasing as $k$ tends to $\infty$. Assume that $\tau_{\infty}$ is the limiting value, i.e., $\lim _{k \rightarrow \infty}$ with $\tau_{\infty} \leq \tau_{e}$ a.s. Here, it is enough to show that $\tau_{\infty}=\infty$ a.s., then $\tau_{e}=\infty$, which prove that $(S(t), I(t), R(t), Z(0)) \in R_{+}^{4}$ a.s., for every $t \geq 0$. We verify that $\tau_{e}=\infty$ a.s to alternately to achieve the remaining derivation. If this not holds, then there will be two constants $T>0$ lies and $\epsilon \in(0,1)$, like

$$
P\left\{\tau_{\infty} \leq T\right\}>\epsilon .
$$

Therefore, integer $k_{0} \leq k_{1}$ will be there as

$$
P\left\{\tau_{k} \leq T\right\} \geq \epsilon \text {, for all } k \geq k_{1} .
$$

Define the following Lyapunov function:

$$
\begin{aligned}
\mathrm{H}= & \left(-C+S-C \frac{\log S}{C}\right)+(I-1-\log I) \\
& +(R-1-\log R)+(Z-1-\log Z) .
\end{aligned}
$$

Applying Itô formula to $H$, and $C$ will be determined later

$$
\begin{aligned}
\mathrm{d} H & (S, I, R, Z) \\
= & {\left[\left(1-\frac{C}{S}\right)\left(\Lambda-\beta S I-\mu_{2} m Z S-\left(\mu_{0}+v\right) S\right)\right.} \\
& \left.+\frac{1}{2} C \eta_{1}{ }^{2}\right] \mathrm{d} t+\left(1-\frac{C}{S}\right) \eta_{1} S \mathrm{~d} B_{1}(t)
\end{aligned}
$$

$$
\begin{aligned}
& +\left[\left(1-\frac{1}{I}\right)\left(\beta S I-\left(\mu_{0}+\gamma_{1}+\mu_{1}\right) I\right)+\frac{1}{2} \eta_{2}^{2}\right] \mathrm{d} t \\
+ & \left(1-\frac{1}{I}\right) \eta_{2} I \mathrm{~d} B_{2}(t) \\
& +\left[\left(1-\frac{1}{R}\right)\left(\gamma_{1} I+\mu_{2} m Z S+v S-\mu_{0} R\right)\right. \\
& \left.+\frac{1}{2} \eta_{3}^{2}\right] \mathrm{d} t+\left(1-\frac{1}{R}\right) \eta_{3} R \mathrm{~d} B_{3}(t) \\
& +\left[\left(1-\frac{1}{Z}\right)\left(\frac{\mathrm{d} I}{1+h I}-a Z\right)\right] \mathrm{d} t, \\
= & \mathrm{LH}(S, I, R, Z) \mathrm{d} t+\eta_{1}(S-C) \mathrm{d} B_{1}(t) \\
& +\eta_{2}(I-1) \mathrm{d} B_{2}(t)+\eta_{3}(R-1) \mathrm{d} B_{3}(t) .
\end{aligned}
$$

In Eq. (8), we define the $L H: R_{+}^{4} \rightarrow R_{+}$by the assertion

$$
\begin{aligned}
& \mathrm{LH}=\left(1-\frac{C}{S}\right)\left(\Lambda-\beta S I-\mu_{2} m Z S-\left(\mu_{0}+v\right) S\right) \\
& +\left(1-\frac{1}{I}\right)\left(\beta S I-\left(\mu_{0}+\gamma_{1}+\mu_{1}\right) I\right) \\
& +\left(1-\frac{1}{R}\right)\left(\gamma_{1} I+\mu_{2} m Z S+v S-\mu_{0} R\right) \\
& +\left(1-\frac{1}{Z}\right)\left(\frac{d I}{1+h I}-a Z\right) \\
& ++\frac{1}{2} C \eta_{1}{ }^{2}+\frac{1}{2} \eta_{2}{ }^{2}+\frac{1}{2} \eta_{3}{ }^{2}, \\
& =\Lambda-\mu_{2} m Z S-\left(v+\mu_{0}\right) S-\frac{C \Lambda}{S}+C \mu_{2} m Z+C \beta I \\
& +C\left(v+\mu_{0}\right)-\left(\mu_{0}+\gamma_{1}+\mu_{1}\right) I-S \beta \\
& +\left(\mu_{0}+\gamma_{1}+\mu_{1}\right)+\gamma_{1} I+S v-R \mu_{0} \\
& +\mu_{2} m Z S-\gamma_{1} \frac{I}{R} \\
& -\frac{v S}{R}+\mu_{0}-\frac{\mu_{2} m Z S}{R}+\frac{\mathrm{d} I}{1+h I} \\
& -a Z-\frac{\mathrm{d} I}{(1+h I) Z}+a+\frac{C \eta_{1}^{2}+\eta_{2}^{2}+\eta_{3}^{2}}{2} \\
& \leq \Lambda+C \beta I-\left(\mu_{0}+\mu_{1}\right) I+C \mu_{2} m Z+C\left(\mu_{0}+v\right) \\
& +\left(\mu_{0}+\gamma_{1}+\mu_{1}\right)+\mu_{0}+\frac{\mathrm{d} I}{1+h I}+a \\
& +\frac{C \eta_{1}^{2}+\eta_{2}^{2}+\eta_{3}^{2}}{2} \\
& \leq \Lambda+\left(C \beta-\left(\mu_{0}+\mu_{1}\right)\right) I+\frac{C \mu_{2} m d \Lambda}{a\left(\mu_{0}+h \Lambda\right)} \\
& +C\left(\mu_{0}+v\right)+2 \mu_{0}+\gamma_{1}+\frac{d}{h}+a \\
& +\frac{C \eta_{1}^{2}+\eta_{2}^{2}+\eta_{3}^{2}}{2}:=K \text {. }
\end{aligned}
$$

Here, $C=\frac{\mu_{0}+\mu_{1}}{\beta}$, such that $C \beta-\left(\mu_{0}+\mu_{1}\right)=0$

$$
\begin{aligned}
& \mathrm{LH}(S, I, R, Z) \\
& \quad \leq \Lambda+\frac{C \mu_{2} m d \Lambda}{a\left(\mu_{0}+h \Lambda\right)}
\end{aligned}
$$




$$
\begin{aligned}
& +C\left(\mu_{0}+v\right)+2 \mu_{0}+\gamma_{1}+\frac{d}{h} \\
& +a+\frac{\frac{\mu_{0}+\mu_{1}}{\beta} \eta_{1}^{2}+\eta_{2}^{2}+\eta_{3}^{2}}{2}:=K .
\end{aligned}
$$

Here, $K$ is a positive constant, so accordingly

$$
\begin{aligned}
& E\left[H(S, R, I)\left(\tau_{k} \wedge \mathrm{T}\right)\right] \\
& \quad \leq \mathrm{H}(Z(0), R(0), I(0), S(0)) \\
& \quad+\mathrm{E}\left[\int_{0}^{\tau_{k} \wedge \mathrm{T}} K \mathrm{~d} t\right], \\
& \leq \mathrm{H}(Z(0), R(0), I(0), S(0))+\mathrm{T} K .
\end{aligned}
$$

Putting $\Omega_{k}=\tau_{k} \leq T, k \geq k_{1}$. Also, from Eq. (6), it could be noted that $P\left(\Omega_{k}\right) \geq \epsilon$. Consider $\omega \in \Omega_{k}$, at least one $S\left(\tau_{k}, \omega\right), I\left(\tau_{k}, \omega\right), R\left(\tau_{k}, \omega\right), Z\left(\tau_{k}, \omega\right)$ exists, which must be equal of $k$ or $\frac{1}{k}$.

Therefore, $\mathrm{H}\left(Z\left(\tau_{k}\right), R\left(\tau_{k}\right), I\left(\tau_{k}\right), S\left(\tau_{k}\right)\right)$ is greater than $-1+k-\log k$ or $\log +\frac{1}{k}+(-1) k$. Moreover

$$
\mathrm{H}(Z, R, I, S) \geq \mathrm{E}(k-1-\log ) \wedge\left(-1+\log k+\frac{1}{k}\right)
$$

Following Eqs. (6) and (11), we obtain:

$$
\begin{aligned}
& \mathrm{H}(Z(0), R(0), I(0), S(0))+\mathrm{TK} \\
& \quad \geq \mathrm{E}\left[1_{\Omega(\omega)} \mathrm{H}\left(Z\left(\tau_{k}\right), R\left(\tau_{k}\right), I\left(\tau_{k}\right), S\left(\tau_{k}\right)\right)\right] \\
& \quad \geq \epsilon\left[(-1+k-\log k) \wedge\left(-1+\log k+\frac{1}{k}\right)\right] .
\end{aligned}
$$

Here, the function $I_{\Omega(\omega)}$ is called the indicating mapping of $\Omega$. Assuming $k \rightarrow \infty$ implies the results contradiction, i.e., TM, which means that $\tau_{\infty}=\infty$ a.s.

Lemma 1 Let any of the starting values $(S(0), I(0)$, $R(0), Z(0)) \in R_{+}^{4}$, have one solution of system (2) on $0 \leq t$ will in $\Gamma$ having probability of 1 .

Proof We know that $N(t)=S(t)+I(t)+R(t)$, so we can obtain

$$
\begin{aligned}
\mathrm{d} N(t)= & \left(\Lambda-\mu_{0} N(t)-\mu_{1} I(t)\right) \mathrm{d} t+\eta_{1} B_{1}(t) \\
& +\eta_{2} B_{2}(t)+\eta_{3} B_{3}(t) \\
\leq & \left(\Lambda-\mu_{0} N(t)\right) \mathrm{d} t+\eta_{1} B_{1}(t) \\
& +\eta_{2} B_{2}(t)+\eta_{3} B_{3}(t) .
\end{aligned}
$$

Integrating Eq. (14) from $0-t$. Thus, we implies that $\frac{\Lambda}{\mu_{0}+\mu_{1}} \leq \frac{\mathrm{d} N(t)}{\mathrm{d} t} \leq \Lambda-\mu_{0} N(t)$; then, we have

$$
\begin{gathered}
\frac{\Lambda}{\mu_{0}+\mu_{1}} \leq \liminf _{t \rightarrow \infty} N(t) \\
\leq \limsup _{t \rightarrow \infty} N(t) \leq \frac{\Lambda}{\mu_{0}}
\end{gathered}
$$

It can be seen from the values of $S, I$, and $R$ that the proposed model (2) is bounded by $\frac{\Lambda}{\mu_{0}}$. Last equation of system (2) implies that $I \leq \frac{\Lambda}{\mu_{0}}$; we get $\limsup _{t \rightarrow \infty} Z \leq$ $\frac{d \Lambda}{a\left(\mu_{0}+h \Lambda\right)}$. Therefore, we get $\Gamma$ is the invariant, positive, and having bounds. After this, all the solution curves lying any where of $R_{+}^{4}$ will lie in $\Gamma$ and having full probabilistic.

\section{Extinction of the disease}

The current section is devoted to discuss the extinction, and therefore, first, we define the basic reproductive parameter which is denoted by $\mathbf{R}_{\mathbf{s}}$ for stochastic model as stated by Eq. (2) and define by

$$
\mathbf{R}_{\mathbf{s}}=\frac{\beta \Lambda}{\mu_{0}\left(\mu_{0}+\gamma_{1}+\mu_{1}+\frac{\eta_{2}^{2}}{2}\right)}
$$

Theorem 2 Let $\left(Z_{t}, R_{t}, I_{t}, S_{t}\right)$ is the root of model (2) with $\left(Z_{0}, R_{0}, I_{0}, S_{0}\right) \in R_{+}^{4}$, if $\mathbf{R}_{\mathbf{s}}<1$, After this, the root of stochastic hepatitis $B$ system (2) satisfies

$$
\begin{aligned}
& \lim _{t \rightarrow \infty}\langle S(t)\rangle=\frac{\Lambda}{\mu_{0}+v} \quad \text { a.s, } \\
& \limsup _{t \rightarrow \infty} \frac{\log I_{t}}{t}<0 \quad \text { a.s, } \\
& \lim _{t \rightarrow \infty}\langle R(t)\rangle=\frac{v \Lambda}{\mu_{0}\left(\mu_{0}+v\right)} \quad \text { a.s, } \\
& \lim _{t \rightarrow \infty}\langle Z(t)\rangle=0 \quad \text { a.s, }
\end{aligned}
$$

namely the disease extinct with probability one.

Proof By directly integrating the proposed system (2), we could obtain the below system of equations

$$
\begin{aligned}
\frac{S(t)-S(0)}{t}= & \Lambda-\beta\langle S(t) I(t)\rangle-\mu_{2} m\langle S(t) Z(t)\rangle \\
& -\left(\mu_{0}+v\right)\langle S(t)\rangle+\frac{\eta_{1}}{t} \int_{0}^{t} S \mathrm{~d} B_{1}(s) \\
\frac{I(t)-I(0)}{t}= & \beta\langle S(t) I(t)\rangle-\left(\mu_{0}+\mu_{1}+\gamma_{1}\right)\langle I(t)\rangle \\
& +\frac{\eta_{2}}{t} \int_{0}^{t} I \mathrm{~d} B_{2}(s), \\
\frac{R(t)-R(0)}{t}= & \mu_{1}\langle I(t)\rangle+\mu_{2} m\langle S(t) Z(t)\rangle-\mu_{0}\langle R(t)\rangle \\
& +v\langle S(t)\rangle+\frac{\eta_{3}}{t} \int_{0}^{t} R \mathrm{~d} B_{3}(s) .
\end{aligned}
$$


One can calculate by Eq. (17), such that

$$
\begin{aligned}
\frac{R(t)}{t}-R(0) & +\frac{S(t)-S(0)}{t}+\frac{I(t)-I(0)}{t} \\
= & -\left(\mu_{0}+\gamma_{1}\right)\langle I(t)\rangle+\Lambda-\mu_{0}\langle S(t)\rangle \\
& -\mu_{0}\langle R(t)\rangle+\frac{\eta_{1}}{t} \int_{0}^{t} S \mathrm{~d} B_{1}(s) \\
& +\frac{\eta_{2}}{t} \int_{0}^{t} I \mathrm{~d} B_{2}(s)+\frac{\eta_{3}}{t} \int_{0}^{t} R \mathrm{~d} B_{3}(s) .
\end{aligned}
$$

Calculation leads to

$$
\langle S(t)\rangle=\frac{\Lambda}{\mu_{0}}-\frac{\mu_{0}+\gamma_{1}}{\mu_{0}}\langle I(t)\rangle-\langle R(t)\rangle+\phi(t),
$$

where

$$
\begin{aligned}
\Phi(t)= & {\left[-\frac{R(t)-R(0)}{t}-\frac{S(t)-S(0)}{t}-\frac{I(t)-I(0)}{t}\right.} \\
& +\frac{\eta_{1}}{t} \int_{0}^{t} S \mathrm{~d} B_{1}(s)+\frac{\eta_{2}}{t} \int_{0}^{t} I \mathrm{~d} B_{2}(s) \\
& \left.+\frac{\eta_{3}}{t} \int_{0}^{t} R \mathrm{~d} B_{3}(s)\right] \frac{1}{\mu_{0}}
\end{aligned}
$$

Utilizing Itô formula to the second equation of system (2), then

$$
\begin{aligned}
\operatorname{dlog} I(t)= & 7\left[\beta S(t)-\left(\mu_{0}+\gamma_{1}+\mu_{1}\right)-\frac{1}{2} \eta_{2}^{2}\right] \mathrm{d} t \\
& +\eta_{2} \mathrm{~d} B_{2}(t) .
\end{aligned}
$$

The integration leads to the following:

$$
\begin{aligned}
\frac{\log I_{t}-\log I_{0}}{t}= & \frac{1}{t} \int_{0}^{t}\left[\beta S-\left(\mu_{0}+\mu_{1}+\gamma_{1}\right)\right. \\
& \left.-\frac{1}{2} \eta_{2}^{2}\right] \mathrm{d} s+\frac{1}{t} \int_{0}^{t} \eta_{2} \mathrm{~d} B_{2}(s) .
\end{aligned}
$$

Following [64], i.e., the large number theorem and local martingles gives

$$
\limsup _{t \rightarrow \infty} \frac{1}{t} \int_{0}^{t} \eta_{i} \mathrm{~d} B_{i}(s)=0
$$

Where $i=\{1,2,3, \ldots\}$, and obviously, $\phi(t)=0$ if we use Eq. (23) and $t \rightarrow \infty$

$$
\begin{aligned}
{[\beta S} & \left.-\left(\mu_{0}+\mu_{1}+\gamma_{1}\right)-\frac{1}{2} \eta_{2}^{2}\right] \\
\leq & \beta\left(\frac{\Lambda}{\mu_{0}}-\frac{\mu_{0}+\mu_{1}}{\mu_{0}}\langle I(t)\rangle-\langle R(t)\rangle+\phi(t)\right) \\
& -\left(\mu_{1}+\mu_{0}+\gamma_{1}\right)-\frac{1}{2} \eta_{2}^{2} \\
& \leq\left(\mu_{1}+\mu_{0}+\gamma_{1}+\frac{\eta_{2}^{2}}{2}\right)\left(\mathbf{R}_{\mathbf{s}}-1\right) .
\end{aligned}
$$

Therefore we obtain

$$
\limsup _{t \rightarrow \infty} \frac{\log I_{t}}{t} \leq\left(\mu_{1}+\mu_{0}+\gamma_{1}+\frac{\eta_{2}^{2}}{2}\right)\left(\mathbf{R}_{\mathbf{s}}-1\right)<0
$$

Denote $\Omega_{1}=\left\{\omega \in \Omega: \lim \sup _{t \rightarrow \infty} I(\omega, t)=0\right\}$. In the view of Eq. (25), we have

$$
P\left(\omega_{1}\right)=1
$$

this implies that at any non-negative $\epsilon_{1}$; there is $T_{1}=$ $T_{1}\left(w, \epsilon_{1}\right)$, so $I(t)<\epsilon_{1}$, a.s, for $T_{1}>t$, then

$$
\begin{aligned}
\mathrm{d} Z(\omega, t)= & {\left[\frac{\mathrm{d} I(\omega, t)}{I+h I(\omega, t)}-a Z(\omega, t)\right] \mathrm{d} t } \\
& \leq\left[\mathrm{d} \epsilon_{1}-a Z(\omega, t)\right] \mathrm{d} t \text { for } \omega \in \Omega_{1}, t \leq T_{1} .
\end{aligned}
$$

Comparison of Theorem [65] gives

$$
\begin{aligned}
Z(\omega, t) \leq & e^{-a t}\left(Z\left(T_{1}\right)+\int_{T_{1}}^{t} \mathrm{~d} \epsilon_{1} \cdot e^{-a s} \mathrm{~d} s\right) \\
& \leq Z\left(T_{1}\right) e^{-\mathrm{d} t}+\frac{\mathrm{d} \epsilon_{1}}{a} \text { for } \omega \in \Omega_{1}, t \leq T_{1} .
\end{aligned}
$$

Then

$$
\limsup _{t \rightarrow \infty} Z(w, t) \leq \frac{\mathrm{d} \epsilon_{1}}{a} \text { for } \omega \in \Omega_{1}, t \leq T_{1}
$$

As $\epsilon_{1}$ is arbitrary, which implies $0 \leq \limsup _{t \rightarrow \infty} Z$. Also, $\lim \sup _{t \rightarrow \infty} Z(\omega, t) \geq 0$. Because of this

$$
\lim _{t \rightarrow \infty}\langle Z(\omega, t)\rangle=0
$$

Let $\Omega_{2}=\omega \in \Omega: \lim \sup _{t \rightarrow \infty} Z(\omega, t)=0 \subset \Omega_{1}$, and then, for $\epsilon_{2} \leq 0$, there is a constant $T_{2}=T_{2}(w, t) \leq T_{1}$ exists and so $Z(t)<\epsilon_{2}$, a.s. for $t>T_{2}$.

Now, put Eqs. (23), (25), and (30) in Eq. (19), we can obtain

$$
\lim _{t \rightarrow \infty}\langle R(t)+S(t)\rangle=\frac{\Lambda}{\mu_{0}} \text { s.a. }
$$

From first equation of model Eq. (17), we have

$$
\begin{aligned}
\frac{S(t)-S(0)}{t}=\Lambda & -\beta\langle S(t) I(t)\rangle-\mu_{2} m\langle S(t) Z(t)\rangle \\
& -\left(\mu_{0}+v\right)\langle S(t)\rangle+\frac{\eta_{1}}{t} \int_{0}^{t} S \mathrm{~d} B_{1}(s),
\end{aligned}
$$

which implies that

$$
\langle S(t)\rangle=\frac{\Lambda}{\left(\mu_{0}+v\right)}-\frac{\beta\langle S(t) I(t)\rangle}{\left(\mu_{0}+v\right)}
$$




$$
\begin{aligned}
& -\frac{\mu_{2} m\langle S(t) Z(t)\rangle}{\left(\mu_{0}+v\right)}-\frac{S(t)-S(0)}{t} \\
& +\frac{\eta_{1}}{t} \int_{0}^{t} S \mathrm{~d} B_{1}(s) ;
\end{aligned}
$$

consequently, Eqs. (23), (25), (30), and (20), we can get

$$
\lim _{t \rightarrow \infty}\langle S(t)\rangle=\frac{\Lambda}{\left(\mu_{0}+v\right)}, \quad \text { s.a. }
$$

Now, we have

$$
\lim _{t \rightarrow \infty}\langle R(t)\rangle=\frac{v \Lambda}{\mu_{0}\left(\mu_{0}+v\right)} \text { a.s. }
$$

The last equation is completion of our proof.

\section{Persistence of the stochastic hepatitis B model}

Now, here, we are going to present the condition for the existence of solution for system (2), while the main work of the on-going part is defined by the below result and some well-known axioms.

Lemma 2 (Strong Law) [34,35] Let $M=\{M\}_{0 \leq t}$ be continuous and real valued along with local martingale which vanish as $t \rightarrow 0$, and then

$$
\begin{aligned}
& \lim _{t \rightarrow \infty}\langle M, M\rangle_{t}=\infty \text {, a.s., } \Rightarrow \lim _{t \rightarrow \infty} \frac{M_{t}}{\langle M, M\rangle_{t}}=0, \text { a.s. } \\
& \lim _{t \rightarrow \infty} \sup \frac{\langle M, M\rangle_{t}}{t}<0, \text { a.s., } \Rightarrow \lim _{t \rightarrow \infty} \frac{M_{t}}{t}=0, \text { a.s. }
\end{aligned}
$$

Lemma 3 [34] Let $(S(t), I(t), R(t), Z(t))$ be the solution of system (2) with $(S(0), I(0), R(0), Z(0)) \in \mathbb{R}_{+}^{4}$, and then, $\lim \sup _{t \rightarrow \infty}(S(t)+I(t)+R(t)+Z(t))<\infty$, a.s. Moreover

$$
\begin{aligned}
& \lim _{t \rightarrow \infty} \frac{S(t)}{t}=0, \quad \lim _{t \rightarrow \infty} \frac{I(t)}{t}=0, \quad \lim _{t \rightarrow \infty} \frac{R(t)}{t}=0, \\
& \lim _{t \rightarrow \infty} \frac{Z(t)}{t}=0 \quad \text { a.s, } \\
& \lim _{t \rightarrow \infty} \frac{\ln S(t)}{t}=0, \quad \lim _{t \rightarrow \infty} \frac{\ln I(t)}{t}=0, \\
& \lim _{t \rightarrow \infty} \frac{\ln R(t)}{t}=0, \quad \lim _{t \rightarrow \infty} \frac{\ln Z(t)}{t}=0 \quad \text { a.s, }
\end{aligned}
$$

and

$$
\begin{array}{ll}
\lim _{t \rightarrow \infty} \frac{\int_{0}^{t} S(u) \mathrm{d} B_{1}(u)}{t}=0, & \lim _{t \rightarrow \infty} \frac{\int_{0}^{t} I(u) \mathrm{d} B_{2}(u)}{t}=0, \\
\lim _{t \rightarrow \infty} \frac{\int_{0}^{t} R(u) \mathrm{d} B_{3}(u)}{t}=0, & \lim _{t \rightarrow \infty} \frac{\int_{0}^{t} Z(u)}{t}=0,
\end{array}
$$

Then, the solution of (2)

$$
\begin{aligned}
& \limsup _{t \rightarrow \infty}(S(t)+I(t)+R(t))=\frac{\Lambda}{\mu_{0}}, \\
& \limsup _{t \rightarrow \infty} Z(t)=\frac{d}{a h} \quad \text { a.s. }
\end{aligned}
$$

Definition 1 [69] The proposed model (2) is said to be persistent, if

$$
\liminf _{t \rightarrow \infty} \frac{1}{t} \int_{0}^{t} I(r) \mathrm{d} r>0 \quad \text { a.s. }
$$

Theorem 3 If $\mathbf{R}_{\mathbf{0}}^{\mathbf{s}}=\frac{\beta \Lambda}{\left(\mu_{0}+v+\frac{\mu_{3} m d}{a h}+\frac{\eta_{1}^{2}}{2}\right)\left(\mu_{0}+\mu_{1}+\gamma_{1}+\frac{\eta_{2}^{2}}{2}\right)}$ then for any initial value $(S(0), I(0), R(0), Z(0)) \in R_{+}^{4}$, the disease $I(t)$ has the axiom

$$
\liminf _{t \rightarrow \infty}\langle I(t)\rangle \geq \frac{\left.2 \Lambda \sqrt{\mathbf{R}_{\mathbf{0}}^{\mathbf{s}}}-1\right)}{c_{1} \beta} \quad \text { a.s. }
$$

where $c_{1}=\frac{\Lambda}{\left(\mu_{0}+v+\frac{\mu_{3} m d}{a h}+\frac{\eta_{1}^{2}}{2}\right)}$. Then, we can say that the disease will prevail if $\mathbf{R}_{\mathbf{0}}^{\mathbf{s}}>1$.

Proof Set

$$
V_{1}=-c_{1} \ln S-c_{2} \ln I,
$$

where $c_{1}$ and $c_{2}$ are constants and we will find later. Applying Itô formula, so we have

$$
\begin{aligned}
\mathrm{d} V_{1}= & \mathcal{L} V_{1}-c_{1} \eta_{1} \mathrm{~d} B_{1}(t)-c_{2} \eta_{2} \mathrm{~d} B_{2}(t), \\
\mathcal{L} V_{1}= & c_{1} \mathcal{L}(-\ln S)+c_{2} \mathcal{L}(-\ln I), \\
= & -c_{1} \frac{\Lambda}{S}+c_{1} \beta I+c_{1} \mu_{3} m Z+c_{1}\left(\mu_{0}+v\right) \\
& +c_{1} \frac{1}{2} \eta_{1}^{2}-c_{2} \beta S+c_{2}\left(\mu_{0}+\mu_{1}+\gamma_{1}\right) \\
& +c_{2} \frac{1}{2} \eta_{2}^{2}, \\
\leq & -c_{1} \frac{\Lambda}{S}-c_{2} \beta S+c_{1}\left(\mu_{0}+v+\frac{\mu_{3} m d}{a h}\right. \\
& \left.+\frac{1}{2} \eta_{1}^{2}\right)+c_{2}\left(\mu_{0}+\mu_{1}+\gamma_{1}\right. \\
& \left.+\frac{1}{2} \eta_{2}^{2}\right)+c_{1} \beta I, \\
\leq & -2 \sqrt{c_{1} c_{2} \Lambda \beta}+c_{1}\left(\mu_{0}+v+\frac{\mu_{3} m d}{a h}\right. \\
& \left.+\frac{1}{2} \eta_{1}^{2}\right)+c_{2}\left(\mu_{0}+\mu_{1}+\gamma_{1}\right. \\
& \left.+\frac{1}{2} \eta_{2}^{2}\right)+c_{1} \beta I .
\end{aligned}
$$

Let 


$$
\begin{aligned}
c_{1}= & \frac{\Lambda}{\left(\mu_{0}+v+\frac{\mu_{3} m d}{a h}+\frac{1}{2} \eta_{1}^{2}\right)}, \\
c_{2}= & \frac{\Lambda}{\left(\mu_{0}+\mu_{1}+\gamma_{1}+\frac{1}{2} \eta_{2}^{2}\right)}, \\
\mathcal{L} V_{1} \leq & -2 \sqrt{\frac{\Lambda^{2} \beta \Lambda}{\left(\mu_{0}+v+\frac{\mu_{3} m d}{a h}+\frac{1}{2} \eta_{1}^{2}\right)\left(\mu_{0}+\mu_{1}+\gamma_{1}+\frac{1}{2} \eta_{2}^{2}\right)}} \\
& +2 \Lambda+c_{1} \beta I, \\
= & -2 \Lambda\left(\sqrt{\frac{\left.\mu_{0}+v+\frac{\mu_{3} m d}{a h}+\frac{1}{2} \eta_{1}^{2}\right)\left(\mu_{0}+\mu_{1}+\gamma_{1}+\frac{1}{2} \eta_{2}^{2}\right)}{\left(\mu_{0}\right)}}\right) \\
& +c_{1} \beta I, \\
= & -2 \Lambda\left(\sqrt{\mathbf{R}_{\mathbf{0}}^{\mathbf{s}}}-1\right)+c_{1} \beta I .
\end{aligned}
$$

Substituting Eqs. (45) into (41), and then integrating both side of the stochastic hepatitis B model (2)

$$
\begin{aligned}
\frac{V_{1}(S(t), I(t))-V_{1}(S(0), I(0))}{t} \\
\leq-2 \Lambda\left(\sqrt{\mathbf{R}_{\mathbf{0}}^{\mathbf{s}}}-1\right)+c_{1} \beta\langle I\rangle-\frac{c_{1} \eta_{1}^{2} B_{1}(t)}{t} \\
\quad-\frac{c_{2} \eta_{2}^{2} B_{2}(t)}{t} \\
=-2 \Lambda\left(\sqrt{\mathbf{R}_{\mathbf{0}}^{\mathbf{s}}}-1\right)+c_{1} \beta\langle I\rangle+\Psi(t),
\end{aligned}
$$

where $\Psi(t)=-\frac{c_{1} \eta_{1}^{2} B_{1}(t)}{t}-\frac{c_{2} \eta_{2}^{2} B_{2}(t)}{t}$. From strong law as stated in Lemma (2), we arrive

$$
\lim _{t \rightarrow \infty} \Psi(t)=0 .
$$

From Eq. (46), we have

$$
\begin{aligned}
\langle I(t)\rangle \geq & \frac{2 \Lambda\left(\sqrt{\mathbf{R}_{\mathbf{0}}^{\mathbf{s}}}-1\right)}{c_{1} \beta}-\frac{1}{c_{1} \beta} \Psi(t) \\
& +\frac{1}{c_{1} \beta}\left(\frac{V_{1}(S(t), I(t))-V_{1}(S(0), I(0))}{t}\right) .
\end{aligned}
$$

According to Lemma (3) and Eq. (47), and the limit superior of Eq. (5), we have

$$
\liminf _{t \rightarrow \infty}\langle I(t)\rangle \geq \frac{2 \Lambda\left(\sqrt{\mathbf{R}_{\mathbf{0}}^{\mathbf{s}}}-1\right)}{c_{1} \beta} \text { a.s. }
$$

This finishes the proof of Theorem (3).

\section{Stationary distribution and ergodicity of the stochastic hepatitis B model}

We follow Hasminskii et al. [66] and will prove the ergodicity, and stationary distribution for the model (2) solution. Let $X(t)$ be Markov process and regular time and homogeneous over $R^{d}$

$$
\mathrm{d} X(t)=b X \mathrm{~d} t+\sum_{r=1}^{k} \sigma_{r}(X) \mathrm{d} B_{r}(t)
$$

The diffusion array is define as follows:

$$
A(x)=\left(a_{i j}(x)\right), \quad a_{i j}(t)=\sum_{r=1}^{k} \sigma_{r}^{i}(t) \sigma_{r}^{j}(x) .
$$

Lemma 4 The Markov process $X(t)$ has ergodic stationary distribution $\Pi(\cdot)$ and unique a bounded open domain $U \subset R^{d}$ with regular boundary $U$ exists and holds the following $[67,68]$ :

1. There is a positive number $\varpi$ will positive, such that $\sum_{i, j=1}^{d} a_{i j}(x) \zeta_{1} \zeta_{2} \geq \varpi|\zeta|^{2}, x \in U, \zeta \in R^{d}$.

2. Positive function $C^{2}$ and mapping $V$ exist: $L V$ is negative $\forall x \in R^{d} \backslash U$. Moreover, assume $\rho(\cdot)$ be a function which is integrable w.r.t $\pi(\cdot)$, and then, for every $x \in R^{d} \backslash U$

$$
P\left\{\lim _{T \rightarrow \infty} \frac{1}{T} \int_{0}^{T} \rho(X(t)) \mathrm{d} t=\int_{R^{d}} \rho(x) \pi(\mathrm{d} x)\right\}=1 .
$$

We present the theorem stated below for the required stationary process as well as ergodicity.

Theorem 4 For the proposed system (2) with $\left(S_{0}, I_{0}\right.$, $\left.R_{0}, Z_{0}\right) \in R_{+}^{4}$, if $\mathbf{R}_{\mathbf{s}}>1$, then the stationary distribution $\pi(\cdot)$ exists, and is ergodic.

Proof We assume $\alpha_{1}$ and $\alpha_{2}$ be numbers and are sufficiently large, such that

$$
\begin{gathered}
U=\left\{\left(x_{1}, x_{2}, x_{3}, x_{4}\right) \in \Gamma \frac{1}{\alpha_{1}} \leq x_{1}, x_{2}, x_{3} \leq \frac{\Lambda}{\mu_{0}}\right. \\
\left.-\frac{1}{\alpha_{1}}, \frac{1}{\alpha_{2}} \leq x_{4} \leq \frac{d \Lambda}{a\left(\mu_{0}+h \Lambda\right)}-\frac{1}{\alpha_{2}}\right\} .
\end{gathered}
$$

It is also to be noted that system (2) can also be written as

$$
\begin{aligned}
d\left[\begin{array}{l}
S \\
I \\
R \\
Z
\end{array}\right] & =\left[\begin{array}{c}
\Lambda-\beta S(t) I(t)-\mu_{2} m Z(t) S(t)-\left(\mu_{0}+v\right) S(t) \\
\beta S(t) I(t)-I(t)\left(+\gamma_{1}+\mu_{1}+\mu_{0}\right) \\
\gamma_{1} I(t)+\mu_{2} m Z(t) S(t)+v S(t)-\mu_{0} R(t) \\
\frac{\mathrm{d} I(t)}{(1+h I(t))}-a Z(t)
\end{array}\right] \mathrm{d} t \\
& +\left[\begin{array}{c}
\eta_{1} S(t) \mathrm{d} B_{1}(t) \\
\eta_{2} I(t) \mathrm{d} B_{2}(t) \\
\eta_{3} R(t) \mathrm{d} B_{3}(t) \\
0
\end{array}\right] .
\end{aligned}
$$


Consequently, the diffusion matrix for the associated problem (2) is as

$$
B=\left[\begin{array}{cccc}
\eta_{1}^{2} S^{2} & 0 & 0 & 0 \\
0 & \eta_{2}^{2} I^{2} & 0 & 0 \\
0 & 0 & \eta_{3}^{2} R^{2} & 0 \\
0 & 0 & 0 & 0
\end{array}\right]
$$

As $U$ is subset of $R_{+}^{4}$ while $\xi \in R_{+}^{4} \backslash\left\{\left(\xi_{1}, \xi_{2}, \xi_{3}, \xi_{4}\right) \in\right.$ $\left.R_{+}^{4}: \xi_{1}=\xi_{2}=\xi_{3}=\xi_{4}\right\}$, then a positive number $C$ exists as

$$
\begin{aligned}
& \sum_{i, j=1}^{4} a_{i j}(S, I, R, Z) \xi_{i} \xi_{j} \\
& \quad=\eta_{1}^{2} S^{2} \xi_{1}^{2}+\eta_{2}^{2} I^{2} \xi_{2}^{2}+\eta_{3}^{2} R^{2} \xi_{3}^{2}+Z^{2} \xi_{4}^{2} \geq C
\end{aligned}
$$

Then, condition (1) of Lemma (4) holds. Let $V_{1}(I)$, $V_{2}(S, I)$ and $V_{3}(S, R, Z)$ be the function for $(S, I, R, Z)$ $\in R_{+}^{4}$ and defined by

$$
\begin{aligned}
V_{1}(I) & =\frac{1}{q} I^{-q}, \\
V_{2}(S, I) & =\frac{1}{q} I^{-q}\left(\frac{\Lambda}{\mu_{0}}-S\right), \\
V_{3}(S, R, Z) & =\frac{1}{S}+R+\frac{1}{Z},
\end{aligned}
$$

where $q$ is positive constant and we will determine it later. Defining a function like

$$
\begin{aligned}
& V(S, I, R, Z)=V_{1}(I)+V_{2}(S, I)+V_{3}(S, R, Z), \\
& V(S, I, R, Z)=\frac{1}{q} I^{-q}+\frac{1}{q} I^{-q}\left(\frac{\Lambda}{\mu_{0}}-S\right) \\
&+\left(\frac{1}{Z}+\frac{1}{S}+R\right) .
\end{aligned}
$$

By the expression Itô and the application of model (2), we obtain

$$
\begin{aligned}
\mathrm{LV}_{1}(I)= & -I^{-q}\left(S \beta-\left(\mu_{0}+\mu_{1}+\gamma_{1}\right)\right)-\frac{1}{2}(q+1) \eta_{2}^{2} I^{-q} \\
= & I^{-q}\left(-\left(\mu_{0}+\mu_{1}+\gamma_{1}+\frac{\eta_{2}^{2}}{2}\right)\left(\mathbf{R}_{\mathbf{s}}-1\right)\right. \\
& \left.-\frac{q}{2} \eta_{2}^{2}\right)+I^{-q} \beta\left(\frac{\Lambda}{\mu_{0}}-S\right) .
\end{aligned}
$$

Then, we compute $\operatorname{LV}_{2}(S, I)$

$$
\begin{aligned}
\operatorname{LV}_{2}(S, I)= & I^{-q}\left(\frac{\Lambda}{\mu_{0}}-S\right)\left(\left(\mu_{0}+\mu_{1}+\gamma_{1}\right)-S \beta\right. \\
& \left.+\frac{1}{2}(q+1) \eta_{2}^{2}\right)-\frac{1}{q} I^{-q} \\
& \left(\Lambda-\beta S I-\mu S \mu_{2} m Z S+\mu_{0} R\right) \\
\leq & I^{-q}\left(\frac{\Lambda}{\mu_{0}+v}-S\right)\left(\mu_{0}+\mu_{1}+\gamma_{1}-\frac{\mu_{0}}{q}\right. \\
& \left.+\frac{1}{2}(q+1) \eta_{2}^{2}\right)+\frac{1}{q} \mu_{2} m Z S I^{-q}+\frac{\beta \Lambda}{q \mu_{0}} I^{-q}
\end{aligned}
$$

For $V_{3}(S, R, Z)$, it implies that

$$
\begin{aligned}
V_{3}(S, R, Z) & =-\frac{\Lambda}{S^{2}}+\frac{\beta I}{S}+\frac{\mu_{0}+v}{S}+\frac{\mu_{2} m Z}{S}-\frac{\eta_{1}^{2}}{S} \\
& +\gamma_{1} I(t)+\mu_{2} m Z(t) S(t)+v S(t) \\
& -\mu_{0} R(t) \frac{d I}{(I+h I) Z^{2}}+\frac{a}{Z} \\
& \leq-\frac{\Lambda}{S^{2}}+\left[\frac{\beta \Lambda}{\mu_{0}}+\mu_{0}+\frac{\mu_{1} m d \Lambda}{a_{\mu}(\mu+h \Lambda)}\right. \\
& \left.-\frac{\mu_{2}^{2} d^{2} \Lambda^{2}}{a^{2}\left(\mu_{0}+h \Lambda\right)^{2}-\frac{e t a_{1}^{2}}{2}}-\frac{\eta_{1}^{2}}{2}\right] \frac{1}{S} \\
& +\frac{\gamma_{1} \Lambda}{\mu_{0}}+\frac{\mu_{2} m d \Lambda^{2}}{a \mu_{0}\left(\mu_{0}+h \Lambda\right)} \\
& +\frac{d \mu_{0} I}{\left(\mu_{0}+h \Lambda\right) Z^{2}} \frac{d}{Z} .
\end{aligned}
$$

Combining (55), (56), and (57), we have

$$
\begin{aligned}
L V & \leq I^{-q}\left(-\left(\mu_{0}+\mu_{1}+\gamma_{1}+\frac{\eta_{2}^{2}}{2}\right)\left(\mathbf{R}_{\mathbf{s}}-1\right)-\frac{q}{2} \eta_{2}^{2}\right) \\
& +I^{-q}\left(\frac{\Lambda}{\mu_{0}+v}-S\right)\left(\mu_{0}+\beta+\mu_{1}+\gamma_{1}-\frac{\mu_{0}}{q}\right. \\
& \left.+\frac{1}{2}(q+1) \eta_{2}^{2}\right)+\frac{1}{q} \mu_{2} m Z S I^{-q}+\frac{\beta \Lambda}{q \mu_{0}} I^{-q} \\
& -\frac{\Lambda}{S^{2}}+\left[\frac{\beta \Lambda}{\mu_{0}}+\mu_{0}+\frac{\mu_{1} m d \Lambda}{a_{\mu}(\mu+h \Lambda)}\right. \\
& \left.+\frac{\mu_{2}^{2} d^{2} \Lambda^{2}}{a^{2}\left(\mu_{0}+h \Lambda\right)^{2}}-\frac{\eta_{1}^{2}}{2}\right] \frac{1}{S} \\
& +\frac{\gamma_{1} \Lambda}{\mu_{0}}+\frac{\mu_{2} m d \Lambda^{2}}{a \mu_{0}\left(\mu_{0}+h \Lambda\right)}+\frac{d \mu_{0} I}{\left(\mu_{0}+h \Lambda\right) Z^{2}}
\end{aligned}
$$

Since $\mathbf{R}_{\mathbf{s}} \geq 0$, and choose sufficiently small, such that

$$
\begin{array}{r}
\left.-\left(\mu_{0}+\mu_{1}+\gamma_{1}+\frac{\eta_{2}^{2}}{2}\right)\left(\mathbf{R}_{\mathbf{s}}-1\right)-\frac{q}{2} \eta_{2}^{2}\right)<0 \\
\mu_{0}+\mu_{1}+\gamma_{1}+\beta-\frac{\mu_{0}}{q}-\frac{1}{2}(1+q) \eta_{2}^{2}<0 .
\end{array}
$$


Likewise, $\frac{\Lambda}{\mu_{0}+\mu_{1}} \leq S+I+R \leq Z \leq \frac{d \Lambda}{a(\mu+h \Lambda)}$, then for $(S, I R, Z) \in \Gamma U$ either $S \leq \frac{1}{\alpha_{1}} I \leq \frac{1}{\alpha_{1}}, R \leq \frac{1}{\alpha_{1}}$ or $Z \leq \frac{1}{\alpha_{2}}$. It is simple from Eq. (58), as for $\alpha_{1}$ or $\alpha_{2}$, sufficiently large $L V \leq-1$ for $(S, I, R, Z) \in \Gamma \backslash U$. Therefore, Lemma (4) holds and the model (2) admits an ergodic invariant distribution $\Pi(\cdot)$, which is unique. Hence, $\left(S_{t}, I_{t}, R_{t}, Z_{t}\right)$ is ergodic and

$$
\begin{gathered}
P\left\{\lim _{t \rightarrow \infty} \frac{1}{t} \int_{0}^{t} \chi\left(S_{s}, I_{s}, R_{s}, Z_{s}\right) \in \Gamma d s\right. \\
\left.=\int_{R^{4}} \chi \Gamma^{\pi}(d x)\right\}=1,
\end{gathered}
$$

where $\chi \Gamma$ is the characteristic function of $\Gamma$.

\section{Numerical simulation of the stochastic hepatitis $B$ model}

Now, in this part, we are presenting some approximate solution by simulation to understand about the theoretical results. For this, we have applied the stochastic Runge-Kutta method to obtain the following discretization transformation of system (2):

$$
\begin{aligned}
S_{i+1}= & S_{i}+\left[\Lambda-\mu_{2} m Z_{i} S_{i}-\beta S_{i} I_{i}-\left(\mu_{0}+v\right) S_{i}\right] \triangle t \\
& +\eta_{1} S_{i} \sqrt{\triangle t} \zeta_{1, i}+\frac{\eta_{1}^{2}}{2} S_{i}\left(\zeta_{1, i}^{2}-1\right) \triangle t \\
I_{i+1}= & I_{i}+\left[\beta S_{i} I_{i}-\left(\mu_{0}+\gamma_{1}+\mu_{1}\right) I_{i}\right] \triangle t \\
& +\eta_{2} I_{i} \sqrt{\triangle t} \zeta_{2, i}+\frac{\eta_{2}^{2}}{2} I_{i}\left(\zeta_{2, i}^{2}-1\right) \triangle t
\end{aligned}
$$

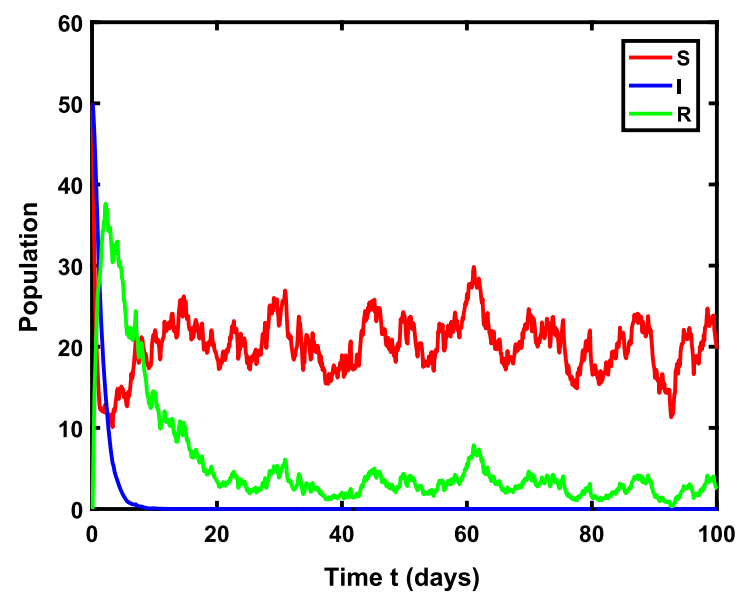

(a) Hepatitis B stochastic System

$$
\begin{aligned}
R_{i+1}= & R_{i}+\left[\left(\gamma_{1} I_{i}+\mu_{2} m Z_{i} S_{i}+v S_{i}-\mu_{0} R_{i}\right)\right] \triangle t \\
& +\eta_{3} R_{i} \sqrt{\triangle t} \zeta_{3, i}+\frac{\eta_{3}^{2}}{2} R_{i}\left(\zeta_{3, i}-1\right) \triangle t, \\
Z_{i+1}= & Z_{i}+\left[{\frac{d I_{i}}{\left(1+h I_{i}\right)}}^{2}-a Z_{i}\right] \triangle t .
\end{aligned}
$$

We perform approximate solution by simulation techniques of the considered hepatitis B stochastic disease system (2) and (1). For this propose, we use the wellknown "stochastic Runge-Kutta method". This analysis verify our analytical findings of the influence of environmental noise and information intervention. It has been found that white noise and information intervention have a big effects on the disease. Now, we are going to assume the numerical value of the parameters with biological feasibility to verify the extinction and the diseases free equilibrium $E_{1}$ result are as, see Fig. 1. Here, we take the noise $\eta_{1}=0.25, \eta_{2}=0.12$, $\eta_{3}=0.525$, and the parameters have the assigned values given as $\Lambda=5.5, \beta=0.015, \mu_{0}=0.4, \gamma_{1}=0.31$, $\mu_{1}=0.4, m=.02, \mu_{2}=0.1, v=0.04, h=0.4, d=0.4$, and $a=0.9$, whereas the initial population sizes will be considered as aforementioned. The result of Theorem 2 shows the behavior of the proposed equation (2) and at the equilibrium point $E_{1}$ before infection is stochastically asymptotically stable. Figure $1 \mathrm{a}$ and $\mathrm{b}$ shows that the stochastic system (2) and the deterministic system (1), respectively, have the same behavior. Both the model's solutions are converging to the disease-free equilibrium point $E_{1}$. This shows that the disease will extinct, i.e., the infection cases will approaches to zero, while there will be always susceptible population.

In a similar fashion, we assume the following parameter value and the intensity of white noise to show the permanence or stationary distribution and endemic equilibrium $E_{2}$, i.e., $\Lambda=5.5, \beta=0.015, \mu_{0}=0.2, \gamma_{1}=$ $0.1, \mu_{1}=0.004, m=0.2, \mu_{2}=0.2, v=0.04, h=0.4$,

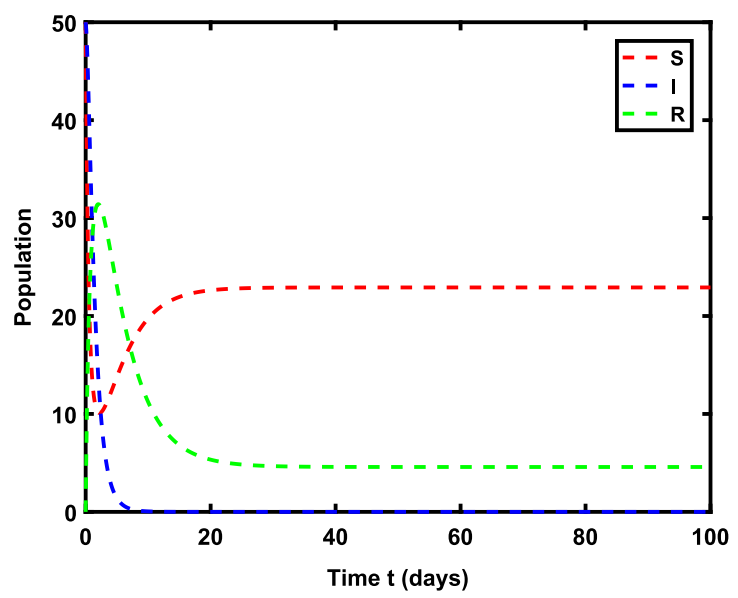

(b) Hepatitis B deterministic system

Fig. 1 The trajectories show the dynamics of $S(t), I(t)$, and $R(t)$ : a represents the stochastic model (2) and b represents its corresponding deterministic model 


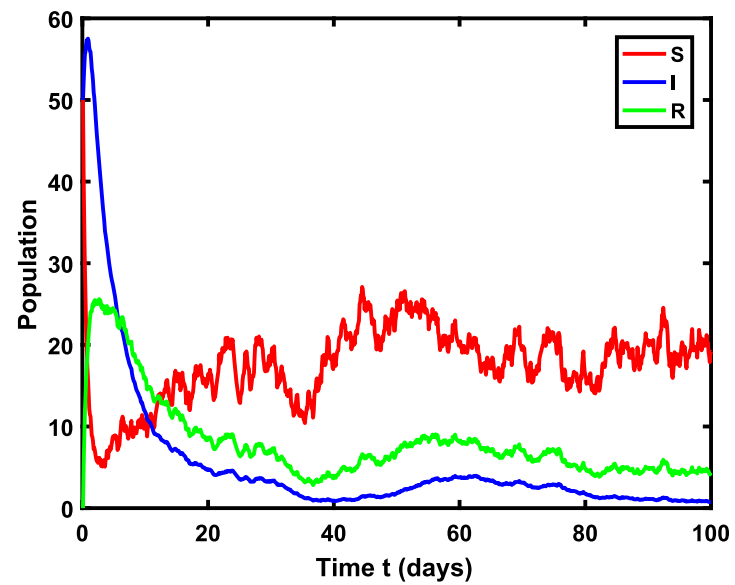

(a) Hepatitis B stochastic System

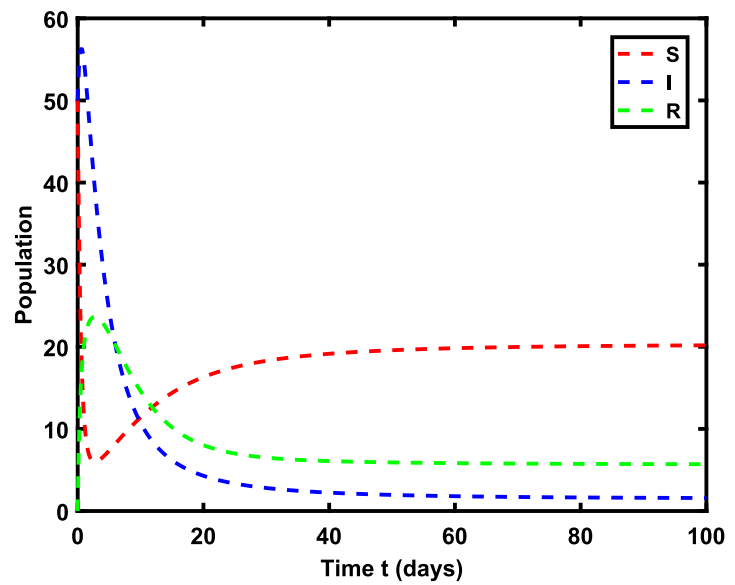

(b) Hepatitis B deterministic system

Fig. 2 The trajectories shows the dynamics of $S(t), I(t)$, and $R(t)$ : a represents the stochastic model (2) and $\mathbf{b}$ represents its corresponding deterministic model

$d=0.2$ and $a=0.9$, and the noise intensities are $\eta_{1}=0.25, \eta_{2}=0.12$, and $\eta_{3}=0.25$, while the initial population sizes will be taken as above. The Theorem 3 axioms are satisfied. Therefore, the stochastic system (2) solutions moves up and down for a more time through the feasible unique disease equilibrium $E_{2}$ of the idealistic system (1), and the mean motion through the disease equilibrium point $E_{2}$ are low because of the small noise waves that the disease come back will exist. Models of both types tend to the disease equilibrium point $E_{2}$ (shown in Fig. $2 \mathrm{a}$ and b). Figure 2 shows the comparison between Eq. (1) and Eq. (2). Once again the three curves in Fig. 3a shows the dynamical behavior of susceptible, infected, and recovered population, which shows that the model maintains the permanence.

Furthermore, for the stationary distribution, to display the effect of the noise intensity on the stationary distribution, we have run the numerical simulation 1000 times and obtain the average extinction time of $S(t)$, $I(t)$, and $R(t)$ at $t=351$, and their distributions are exhibited in Fig. 3 (see for more details Fig. 3a-c). Then, the conditions of Theorem 4 are satisfied.

\section{Variable-order fractional network}

Herein, we aim to answer this question: How can we model the impact of the disease rate in different societies. In other words, we want to embed the interaction between different communities in the modeling of the disease. For instance, the relationships and travels between two countries will definitely affect the rate of diseases in both of them. To the best of our knowledge, up to now, no study has investigated this matter in the modeling of hepatitis B. We proposed to consider the model of the disease as neurons affected by each other in a network. Also, the fractions calculus, which is the generalized form of integer one and provides more accurate results, is considered for the disease modeling. To construct a network of stochastic hepatitis B model, we couple $N$ numbers of these models through the nearest-neighbor method. It is supposed that the interaction between neurons is through the susceptible components, i.e., the coupling is made on the variable $S$. Consequently, the equations of the network is described as

$$
\begin{aligned}
\frac{d^{q(t)} S_{i}}{d t^{q(t)}=} & \Lambda-\beta S_{i}(t) I_{i}(t)-\mu_{2} m Z_{i}(t) S_{i}(t) \\
& -\left(v+\mu_{0}\right) S_{i}(t) \\
& +\frac{l}{2 p} \sum_{j=i-p}^{i+p}\left(S_{j}-S_{i}\right) \\
\frac{d^{q(t)} I_{i}}{d t^{q(t)}}= & \beta I_{i}(t) S_{i}(t)-\left(\mu_{0}+\gamma_{1}+\mu_{1}\right) I_{i}(t) \\
\frac{d^{q(t)} R_{i}}{d t^{q(t)}}= & \gamma_{1} I_{i}+\mu_{2} m Z_{i}(t) S_{i}(t)+v S_{i}(t)-\mu_{0} R_{i}(t) \\
\frac{d^{q(t)} Z_{i}}{d t^{q(t)}}= & \frac{d I_{i}(t)}{\left(1+h I_{i}(t)\right)}-a Z_{i}(t)
\end{aligned}
$$

where $q(t)$ indicates the time-varying fractional-order derivative. $l$ is the coupling strength, $P$ is the number of nearest neighbors on each side, and $N$ denotes the number of systems in the network. Also, similar to (2), using the standard Brownian motions, i.e., $B_{1}(t), B_{2}(t)$, and $B_{3}(t)$ along with the intensities $h_{1}, h_{2}$ and $h_{3}$, we can propose the type of deterministic model (1) in stochastic style as follows:

$$
\begin{aligned}
d^{q(t)} S_{i}= & {\left[\Lambda-\beta S_{i}(t) I_{i}(t)-\mu_{2} m Z_{i}(t) S_{i}(t)\right.} \\
& \left.-\left(v+\mu_{0}\right) S_{i}(t)+\frac{l}{2 p} \sum_{j=i-p}^{i+p}\left(S_{j}-S_{i}\right)\right] d t^{q(t)} \\
& +\eta_{1} S_{i}(t) d B_{1}(t)
\end{aligned}
$$




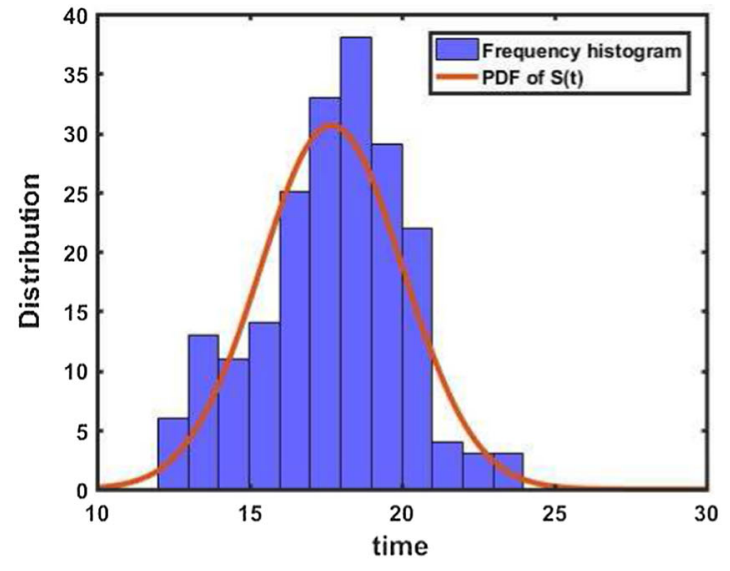

(a) S-Susceptible

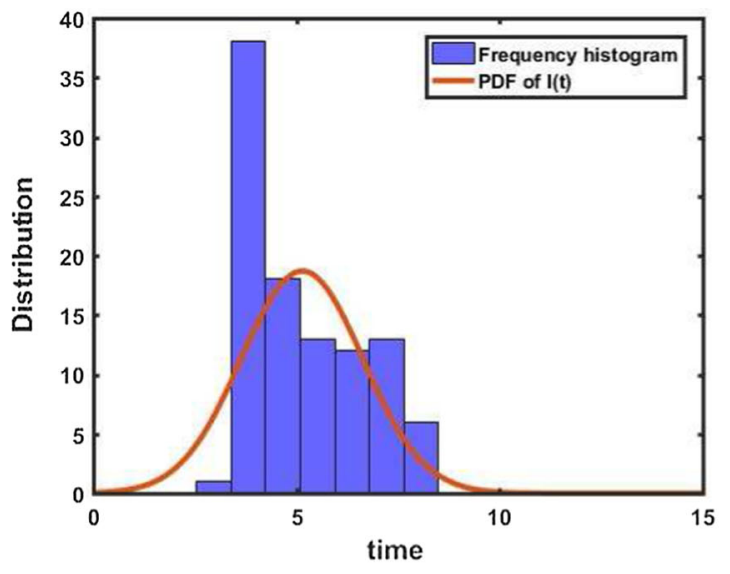

(b) I-Infected

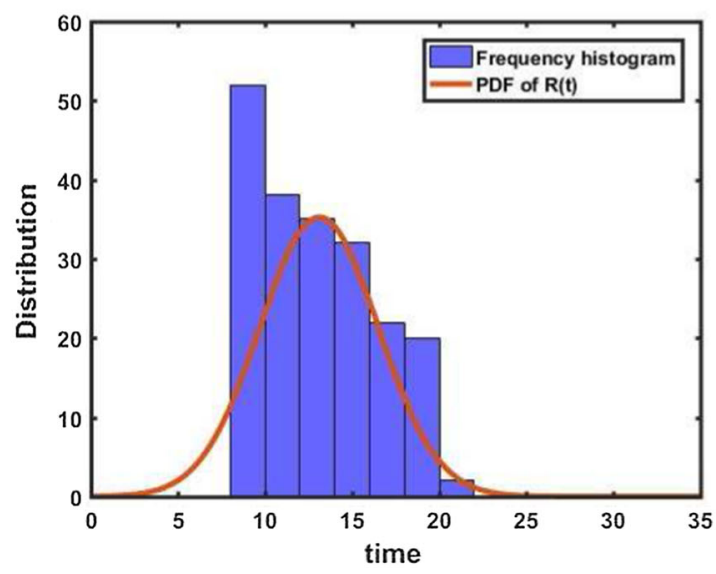

(c) R-Recovered

Fig. 3 Frequency histograms for $S(t), I(t)$, and $R(t)$ under the noise intensities $\eta_{1}=0.25, \eta_{2}=0.12$, and $\eta_{3}=0.25$ of model (2)

$$
\begin{aligned}
\frac{d^{q(t)} I_{i}}{d t^{q(t)}=} & {\left[\beta I_{i}(t) S_{i}(t)-\left(\mu_{0}+\gamma_{1}+\mu_{1}\right) I_{i}(t)\right] d t^{q(t)} } \\
& +\eta_{2} I_{i}(t) d B_{2}(t) \\
\frac{d^{q(t)} R_{i}}{d t^{q(t)}=} & {\left[\gamma_{1} I_{i}+\mu_{2} m Z_{i}(t) S_{i}(t)+v S_{i}(t)-\mu_{0} R_{i}(t)\right] d t^{q(t)} } \\
& +\eta_{3} R_{i}(t) d B_{3}(t) \\
\frac{d^{q(t)} Z_{i}}{d t^{q(t)}}= & {\left[\frac{d I_{i}(t)}{\left(1+h I_{i}(t)\right)}-a Z_{i}(t)\right] d t^{q(t)} }
\end{aligned}
$$

\section{Conclusion}

We investigated the dynamics of hepatitis $\mathrm{B}$ via a stochastic SIR model by incorporating environmental noise and information intervention. We found that white noise and information intervention have considerable effects on the disease. From the dynamics, we noted that it plays a crucial role in the disease spreading and prevalence. We particularly proved the existence analysis and uniqueness analysis and showed that the proposed problem is well posed. We also defined the stochastic reproductive number by $\mathbf{R}_{\mathbf{s}}$ for the problem (2) to discuss the analysis of extinction, persistence, and stationary distribution. On behalf of threshold quantity $\mathbf{R}_{\mathbf{s}}$, we proved that the hepatitis B could be eradicated if the value of $\mathbf{R}_{\mathbf{s}}$ is less than unity, see Theorem (2), and this may reduce the maximum value of infected cases population (see Fig. 1). Moreover, for the stochastic model (2), we have obtained sufficient conditions for the disease prevails of a unique stationary distribution; see Theorem (4). Therefore, the information intervention may reduce the maximum value of infected cases. At the end, all the theoretical findings are justified with 
Fig. 4 The trajectories show the dynamics of neuron (62) in the proposed network: a represents the stochastic model and $\mathbf{b}$ represents its corresponding deterministic model

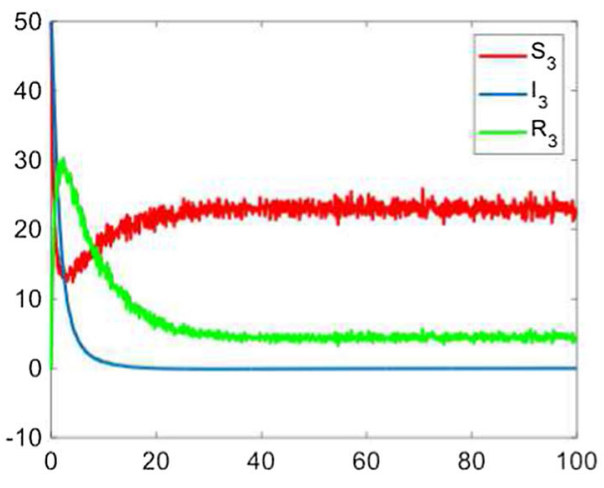

(a) Hepatitis B stochastic System

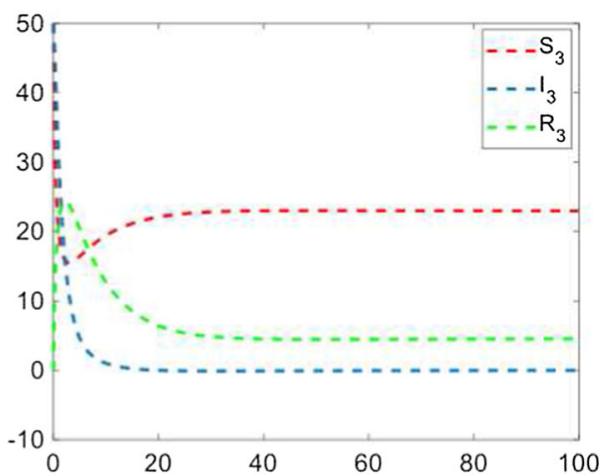

(b) Hepatitis B deterministic system the help of simulation analysis via the stochastic RungeKutta method. Then, as a new approach, a variableorder fractional network of the stochastic hepatitis B model is introduced to consider the effects of different societies on each other. It was supposed that populations are jointed through the nearest-neighbor method. In a feature work, we will investigate the optimal control of the proposed variable-order network.

Acknowledgements This work was supported by the National Natural Science Foundation of P. R. China (nos. 11971493 and 12071491), Fundamental Research Funds for the central Universities, Sun Yat-sen University (3400031610293) (Grant no. 20lgpy137), and the Taif University Researchers Supporting Project number (TURSP-2020/77), Taif University, Taif, Saudi Arabia.

Data Availability Statement Data sharing is not applicable to this article as no new data were created or analyzed in this study.

\section{References}

1. V. Wiwanitkit, B. Shi, S. Xia, G.-J. Yang, X.-N. Zhou, J. Liu, Research priorities in modeling the transmission risks of H7N9 bird flu. Infect. Dis. Poverty 2(1), 17 (2013)

2. World Health Organization. Coronavirus disease 2019 (COVID-19): situation report, p. 82 (2020)

3. B.R. Bloom, T. Godal, Selective primary health care: strategies for control of disease in the developing world. V. Leprosy. Rev. Infect. Dis. 5(4), 765-780 (1983). (Kindly provide necessary detils for the refs. [2, $6,13,43$, if possible.)

4. K.E.F. Watt, The use of mathematics and computers to determine optimal strategy and tactics for a given insect pest control problem. Can. Entomol. 96(1-2), 202-220 (1964)

5. S. Zhao, X. Zhiyi, L. Ying, A mathematical model of hepatitis B virus transmission and its application for vaccination strategy in China. Int. J. Epidemiol. 29(4), $744-752(2000)$
6. K.C. Stafford. Tick Management Handbook; an integrated guide for homeowners, pest control operators, and public health officials for the prevention of tickassociated disease (2007)

7. C.A. Bradley, H. Rolka, D. Walker, J. Loonsk, BioSense: implementation of a national early event detection cind situational awareness system. Morb. Mortal. Week. Rep. MMWR 2005, 5 (2005)

8. W. Zhou, Y. Xiao, J.M. Heffernan, Optimal media reporting intensity on mitigating spread of an emerging infectious disease. PLoS ONE 14(3), 0213898 (2019)

9. Y. Xiao, S. Tang, W. Jianhong, Media impact switching surface during an infectious disease outbreak. Sci. Rep. 5, 7838 (2015)

10. Y. Chen, F. Sun, X. Hu, Y. Liu, A qualitative analysis of the avian influenza (H7N9) model with the influence of media coverage. Basic Sci. J. Textile Univ. 28(4), 408-412 (2015)

11. G. Pennycook, J. McPhetres, Y. Zhang, J.G. Lu, D.G. Rand, Fighting COVID-19 misinformation on social media: experimental evidence for a scalable accuracynudge intervention. Psychol. Sci. 31(7), 770-780 (2020)

12. S. Eubank, I. Eckstrand, B. Lewis, S. Venkatramanan, M. Marathe, C.L. Barrett, Commentary on Ferguson, et al., impact of non-pharmaceutical interventions (NPIs) to reduce COVID-19 mortality and healthcare demand. Bull. Math. Biol. 82, 1-7 (2020)

13. World Health Organization. Guidelines for controlling and monitoring the tobacco epidemic. World Health Organization (1998)

14. B.C. Sirois, M.M. Burg, Negative emotion and coronary heart disease: A review. Behav. Modif. 27(1), 83-102 (2003)

15. D.N. Pamukoff, B. Pietrosimone, M.D. Lewek, E.D. Ryan, P.S. Weinhold, D.R. Lee, J.T. Blackburn, Immediate effect of vibratory stimuli on quadriceps function in healthy adults. Muscle Nerve 54(3), 469-478 (2016)

16. R.T. Alqahtani, A. Yusuf, R.P. Agarwal, Mathematical analysis of oxygen uptake rate in continuous process under caputo derivative. Mathematics 9(6), 675 (2021)

17. T.H. Zhao, O. Castillo, H. Jahanshah, A fuzzy-based strategy to suppress the novel coronavirus (2019NCOV) massive outbreak. Appl. Comput. Math. 20(1), 160-76 (2021) 
18. A. Yusuf, B. Acay, U.T. Mustapha, M. Inc, D. Baleanu, Mathematical modeling of pine wilt disease with Caputo fractional operator. Chaos Soliton. Fract. 143, 110569 (2021)

19. B. Acay, A. Khan, A. Yusuf, Fractional methicillinresistant Staphylococcus aureus infection model under Caputo operator. J. Appl. Math. Comput. 2021, 1-29 (2021)

20. T. Khan, G. Zaman, Classification of different hepatitis B infected individuals with saturated incidence rate. Springerplus 2016, 5 (2016)

21. A. Din, Y. Li, T. Khan, G. Zaman, Mathematical analysis of spread and control of the novel corona virus (COVID-19) in China. Chaos Soliton. Fract. 2020, $110286(2020)$

22. I. Ahmed, G.U. Modu, A. Yusuf, P. Kumam, I. Yusuf, A mathematical model of Coronavirus Disease (COVID19) containing asymptomatic and symptomatic classes. Result. Phys. 21, 103776 (2021)

23. A. Din, A. Khan, D. Baleanu, Stationary distribution and extinction of stochastic coronavirus (COVID-19) epidemic model. Chaos Soliton. Fract. 2020, 110036 (2020)

24. T. Khan, A. Khan, G. Zaman, The extinction and persistence of the stochastic hepatitis B epidemic model. Chaos Soliton. Fract. 108, 123-128 (2018)

25. T. Feng, Z. Qiu, X. Meng, Dynamics of a stochastic hepatitis c virus system with host immunity. Discrete Contin. Dyn. Syst.-B 24(12), 6367 (2019)

26. A. Din, Y. Li, Controlling heroin addiction via agestructured modeling. Adv. Differ. Equ. 2020(1), 1-17 (2020)

27. A. Khan, G. Zaman, Optimal control strategy of SEIR endemic model with continuous age-structure in the exposed and infectious classes. Optim. Control Appl. Methods 39(5), 1716-1727 (2018)

28. A.-K. Alomari, T. Abdeljawad, D. Baleanu, K.M. Saad, Q.M. Al-Mdallal, Numerical solutions of fractional parabolic equations with generalized Mittag-Leffler kernels. Numer. Methods Part. Differ. Equ. 2020, 2 (2020)

29. K.M. Saad, M. Alqhtani, J.F. Gómez-Aguilar, Fractalfractional study of the hepatitis $\mathrm{C}$ virus infection model. Result. Phys. 19, 103555 (2020)

30. H.M. Srivastava, K.M. Saad, M.M. Khader, An efficient spectral collocation method for the dynamic simulation of the fractional epidemiological model of the Ebola virus. Chaos Soliton. Fract. 140, 110174 (2020)

31. N. Dalal, D. Greenhalgh, X. Mao, A stochastic model for internal HIV dynamics. J. Math. Anal. Appl. 341, 1084-101 (2008)

32. A. Lahrouz, L. Omari, Extinction and stationary distribution of a stochastic SIRS epidemic model with nonlinear incidence. Stat. Prob. Lett. 83, 960-8 (2013)

33. T. Khan, I.H. Jung, G. Zaman, A stochastic model for the transmission dynamics of hepatitis B virus. J. Biol. Dyn. 13(1), 328-344 (2019)

34. Y. Hou, W. Zhang, S. Yuan, Survival and stationary distribution of a SIR epidemic model with stochastic perturbations. Appl. Math. Comput. 244, 118-31 (2014)

35. Q. Lu, Stability of SIRS system with random perturbations. Phys. A 388, 3677-86 (2009)

36. C. Ji, D. Jiang, Threshold behaviour of a stochastic SIR model. Appl. Math. Model. 38, 5067-79 (2014)
37. T. Zhang, K. Wang, X. Zhang, Modeling and analyzing the transmission dynamics of HBV epidemic in Xinjiang, China. PLoS ONE 10, e0138765 (2015)

38. J. Mann, M. Roberts, Modelling the epidemiology of hepatitis B in New Zealand. J. Theor. Biol. 269(1), 266$72(2011)$

39. A. Din, Y. Li, Q. Liu, Viral dynamics and control of hepatitisB virus(HBV) using an epidemic model. Alex. Eng. J. 59(2), 667-679 (2020)

40. T. Khan, G. Zaman, M.I. Chohan, The transmission dynamic and optimal control of acute and chronic hepatitis B. J. Biol. Dyn. 11(1), 172-89 (2017)

41. H. Joshi, S. Lenhart, K. Albright, K. Gipson, Modeling the effect of information campaigns on the HIV epidemic in Uganda. Math. Biosci. Eng. 5(4), 757 (2008)

42. B.J. Buonomo, A. d'Onofrio, D. Lacitignola, Modeling of pseudo-rational exemption to vaccination for SEIR diseases. J. Math. Anal. Appl. 404(2), 385-398 (2013)

43. H.R. Joshi, S. Lenhart, S. Hota, F.B. Augusto. Optimal control of an SIR model with changing behavior through an education campaign (2015)

44. A. Din, Y. Li, T. Khan, K. Anwar, G. Zaman, Stochastic dynamics of hepatitis B epidemics. Result. Phys. 20, $103730(2021)$

45. S. Bekiros, H. Jahanshahi, F. Bezzina, A.A. Aly, A novel fuzzy mixed optimal controller for hyperchaotic financial systems. Chaos Soliton. Fract. 146, 110878 (2021)

46. J.-F. Li, H. Jahanshahi, S. Kacar, Y.-M. Chu, J.F. Gómez-Aguilar, N.D. Alotaibi, K.H. Alharbi, On the variable-order fractional memristor oscillator: data security applications and synchronization using a type- 2 fuzzy disturbance observer-based robust control. Chaos Soliton. Fract. 145, 110681 (2021)

47. Y.-L. Wang, H. Jahanshahi, S. Bekiros, F. Bezzina, Y.-M. Chu, A.A. Aly, Deep recurrent neural networks with finite-time terminal sliding mode control for a chaotic fractional-order financial system with market confidence. Chaos Soliton. Fract. 146, 110881 (2021)

48. H. Jahanshahi, D. Chen, Y.-M. Chu, J.F. GómezAguilar, A.A. Aly, Enhancement of the performance of nonlinear vibration energy harvesters by exploiting secondary resonances in multi-frequency excitations. Eur. Phys. J. Plus 136(3), 1-22 (2021)

49. H. Jahanshahi, S.S. Sajjadi, S. Bekiros, A.A. Aly, On the development of variable-order fractional hyperchaotic economic system with a nonlinear model predictive controller. Chaos Soliton. Fract. 144, 110698 (2021)

50. H. Jahanshahi, J.M. Munoz-Pacheco, S. Bekiros, N.D. Alotaibi, A fractional-order SIRD model with timedependent memory indexes for encompassing the multifractional characteristics of the COVID-19. Chaos Soliton. Fract. 143, 110632 (2021)

51. H. Jahanshahi, O. Orozco-López, M. Munoz-Pacheco, N.D. Alotaibi, C. Volos, Z. Wang, R. Sevilla-Escoboza, Y.-M. Chu, Simulation and experimental validation of a non-equilibrium chaotic system. Chaos Soliton. Fract. 143, 110539 (2021)

52. H. Jahanshahi, K. Shanazari, M. Mesrizadeh, S. Soradi-Zeid, J.F. Gómez-Aguilar, Numerical analysis of Galerkin meshless method for parabolic equations of tumor angiogenesis problem. Eur. Phys. J. Plus 135(11), 1-23 (2020) 
53. H. Jahanshahi, A. Yousefpour, J.M. Munoz-Pacheco, S. Kacar, V.-T. Pham, F.E. Alsaadi, A new fractionalorder hyperchaotic memristor oscillator: Dynamic analysis, robust adaptive synchronization, and its application to voice encryption. Appl. Math. Comput. 383, $125310(2020)$

54. H. Jahanshahi, A. Yousefpour, J.M. Munoz-Pacheco, I. Moroz, Z. Wei, O. Castillo, A new multi-stable fractional-order four-dimensional system with selfexcited and hidden chaotic attractors: Dynamic analysis and adaptive synchronization using a novel fuzzy adaptive sliding mode control method. Appl. Soft Comput. 87, 105943 (2020)

55. H. Jahanshahi, A. Yousefpour, Z. Wei, R. Alcaraz, S. Bekiros, A financial hyperchaotic system with coexisting attractors: Dynamic investigation, entropy analysis, control and synchronization. Chaos Soliton. Fract. 126, 66-77 (2018)

56. H. Jahanshahi, K. Rajagopal, A. Akgul, N.N. Sari, H. Namazi, S. Jafari, Complete analysis and engineering applications of a megastable nonlinear oscillator. Int. J. Non-Linear Mech. 107, 126-136 (2018)

57. Q. Han, D. Jiang, C. Ji, Analysis of a delayed stochastic predator-prey model in a polluted environment. Appl. Math. Model. 38(13), 3067-3080 (2014)

58. A. Din, Y. Li, Stationary distribution extinction and optimal control for the stochastic hepatitis B epidemic model with partial immunity. Phys. Scr. 2021, 5 (2021)

59. Y. Ding, X. Min, H. Liangjian, Asymptotic behavior and stability of a stochastic model for AIDS transmission. Appl. Math. Comput. 204(1), 99-108 (2008)
60. J.R. Beddington, R.M. May, Harvesting natural populations in a randomly fluctuating environment. Science 197(4302), 463-465 (1977)

61. A. Din, Y. Li, A. Yusuf, Delayed hepatitis B epidemic model with stochastic analysis. Chaos Soliton. Fract. 146, 110839 (2021)

62. L.J.S. Allen, B.M. Bolker, Y. Lou, A.L. Nevai, Asymptotic profiles of the steady states for an SIS epidemic reaction-diffusion model. Discrete Contin. Dyn. Syst.-A 21(1), 1 (2008)

63. K. Bao, Q. Zhang, Stationary distribution and extinction of a stochastic SIRS epidemic model with information intervention. Adv. Differ. Equ. 2017(1), 352 (2017)

64. X. Mao, Stochastic Differential Equations and Applications (Elsevier, Berlin, 2007)

65. P.E. Ikeda, N.S. Watanabe, Stochastic Differential Equations and Diffusion Processes North-Holland Mathematical Library, 24 (North-Holland Publ. Co., Amsterdam, New York, 1981)

66. R. Schulz, R.Z. Has' minskii, Stochastic stability of differential equations. Mechanics: analysis 7. Alphen aan den Rijn, Sijthoff and Noordhoff 1980. 360 100.00. 50.00. ISBN 90-286-0100-7. ZaMM 62(2), 137-138 (1982)

67. J.V. Scheidt, T.C. Gard, Introduction to Stochastic Differential Equations (Marcel Dekker Inc, New YorkBasel, 1988)

68. G. Strang, Linear Algebra and Its Applications (Thomson Learning Inc, London, 1988)

69. Y. Zhao, D. Jiang, D. O'Regan, The extinction and persistence of the stochastic SIS epidemic model with vaccination. Phys. A 392(20), 4916-4927 (2013) 